\title{
Multi-objective optimization of multi-echelon supply chain networks with uncertain product demands and prices
}

\author{
Cheng-Liang Chen*, Wen-Cheng Lee \\ Department of Chemical Engineering, National Taiwan University, Taipei 10617, Taiwan, ROC
}

\begin{abstract}
A multi-product, multi-stage, and multi-period scheduling model is proposed in this paper to deal with multiple incommensurable goals for a multi-echelon supply chain network with uncertain market demands and product prices. The uncertain market demands are modeled as a number of discrete scenarios with known probabilities, and the fuzzy sets are used for describing the sellers' and buyers' incompatible preference on product prices. The supply chain scheduling model is constructed as a mixed-integer nonlinear programming problem to satisfy several conflict objectives, such as fair profit distribution among all participants, safe inventory levels, maximum customer service levels, and robustness of decision to uncertain product demands, therein the compromised preference levels on product prices from the sellers and buyers point of view are simultaneously taken into account. The inclusion of robustness measures as part of objectives can significantly reduce the variability of objective values to product demand uncertainties. For purpose that a compensatory solution among all participants of the supply chain can be achieved, a two-phase fuzzy decision-making method is presented and, by means of application of it to a numerical example, proved effective in providing a compromised solution in an uncertain multi-echelon supply chain network.
\end{abstract}

(C) 2003 Elsevier Ltd. All rights reserved.

Keywords: Supply chain management; Uncertainty; Robustness; Multiple objectives; Mixed-integer nonlinear programming; Fuzzy optimization

\section{Introduction}

Industries around the world are now all rushing the territory of globalization and specialization. Cooperating with good strategic partners is the sure way to tackle the potential problems arising from competition. Companies can achieve the optimum operating efficiency by working with other companies through communication and specialization, which evolve a new type of relationship, the supply chain relationship, among these companies and further foster a new concept in management: the supply chain management concept. A great variety of companies, those in chemical industry included, can also take advantage of this novel management scheme. Therefore, many researchers in process systems engineering (PSE) society devote themselves to this interesting field (Applequist, Pekny, \& Reklaitis, 2000; Bose \& Pekny, 2000; Chen, Wang, \& Lee, 2003; Garcia-Flores, Wang, \& Goltz, 2000; Gupta \& Maranas, 2000; Gupta, Maranas, \& McDonaldet, 2000; Perea-Lopez,

\footnotetext{
* Corresponding author. +886-2-23636194; fax: +886-2-23623040.

E-mail address: CCL@ntu.edu.tw (C.-L. Chen).
}

Grossmann, \& Ydstie, 2000; Pinto, Joly, \& Moro, 2000; Zhou, Cheng, \& Hua, 2000; to name a few).

In traditional supply chain management, the focus of the integration of supply chain network is usually on single objective, minimum cost or maximum profit. For example, Gjerdrum, Shah, and Papageorgiouet (2001) proposed a mixed-integer linear programming model for a production and distribution planning problem and solve the fair profit distribution problem by using the Nash-type model as the single objective function. However, there are no design tasks that are single objective problems. The design/planning/ scheduling projects are usually involving trade-offs among different incompatible goals (Cheng, Subrahmanian, \& Westerberg, 2003). Recently, a multi-objective production and distribution-scheduling scheme for a supply chain system is formulated by Chen et al. (2003). In this method, in addition to maximizing profit for the entire system, fair profit distribution among all members, customer service levels, and safe inventory levels are taken into account simultaneously. All the problem parameters are deterministically known in the model. In practice, however, this is rarely the case as it is usually difficult to foretell prices of chemicals, market demands, and availabilities of raw materials, etc., in a precise fashion (Liu \& Sahinidis, 1997). 
A number of works have devoted to studying supply chain management under uncertain environments. For example, Gupta and Maranas (2000), Gupta et al. (2003) incorporate the uncertain demand via a normal probability function and propose a two-stage solution framework. A generalization to handle multi-period and multi-customer problems is recently proposed (Gupta \& Maranas, 2003). Tsiakis et al. (2001) use scenario planning approach to describe demand uncertainties. Therein a number of demand scenarios with assigned non-zero probabilities is used as discrete stochastic demand quantities. All scenarios are simultaneously taken into account in the supply chain network design. However, the robustness of decision to uncertain product demands is not considered in these studies. Due to the potential of dealing with linguistic expressions and uncertain issues (Zadeh, 1965; Petrovic, Roy, \& Petrovic, 1998) use fuzzy sets to handle uncertain demands and external raw material problems, and further considering uncertain supply deliveries in a later work (Petrovic, Roy, \& Petrovic, 1999). Giannoccaro, Pontrandolfo, and Scozzi (2003) also apply fuzzy sets theory to model the uncertainties associated with both market demand and inventory costs. The product price, despite with their obvious negotiable and uncertain characteristics in real businesses, seems seldom to be taken into account as a source of uncertainty in previous works. Instead, the product prices at selling points are usually treated as determined parameters.

In this paper, we incorporate two kinds of uncertainties including the market demands and product prices. The scenario-based approach will be adopted for modeling the uncertain market demands, and the product prices will be taken as fuzzy variables where the incompatible preference on prices for different members are handled simultaneously. The whole supply chain scheduling model would turn into a mixed-integer nonlinear programming (MINLP) problem ultimately. The compromised solution for ensuring fair profit distribution, safe inventory levels, maximum customer service levels, decision robustness to uncertain product de- mands, and simultaneously considering incompatible preference of product prices for all participants will be determined by applying the fuzzy multi-objective optimization method.

In the rest of this article, the problem statement and assumptions are outlined in Section 2. The considered uncertain issues in supply chain scheduling are described in Section 3. The formulation of a production and distribution-scheduling model is set out in Section 4. The procedure for grouping the scenario-dependent multiple conflict objectives and uncertain fuzzy product prices into a scalar one using the fuzzy sets concept is presented in Section 5. The contents of a numerical example, used to demonstrate the usefulness of the proposed method, are given in Section 6. Finally, some concluding remarks are drawn in Section 7.

\section{Problem description}

A general supply chain that consists of three different levels of enterprises is considered and showed in Fig. 1 (Chen et al., 2003): the first level enterprise is retailer or market from which the products are sold to customer under the conditions subject to a given low bound of customer service; the second level enterprise is distribution center (DC) or warehouse using different type of transport capacity to deliver products from plant side to retailer side; and the third level enterprise is plant or manufacturer that batch-manufactures one product at one period. The fixed manufacture/idle costs are also employed: on one hand, if the production line is changed over to manufacture another product, manufacture cost would be remained fixed; on the other hand, if the production line is set up to manufacture one specific product but actually is idle, the idle cost, also fixed. Furthermore, the plant has options of manufacturing in regular time or overtime to satisfy the customer demand. To simplify the problem here, we do not consider the problem of purchase and inventory of the raw material in plants nor incorporate

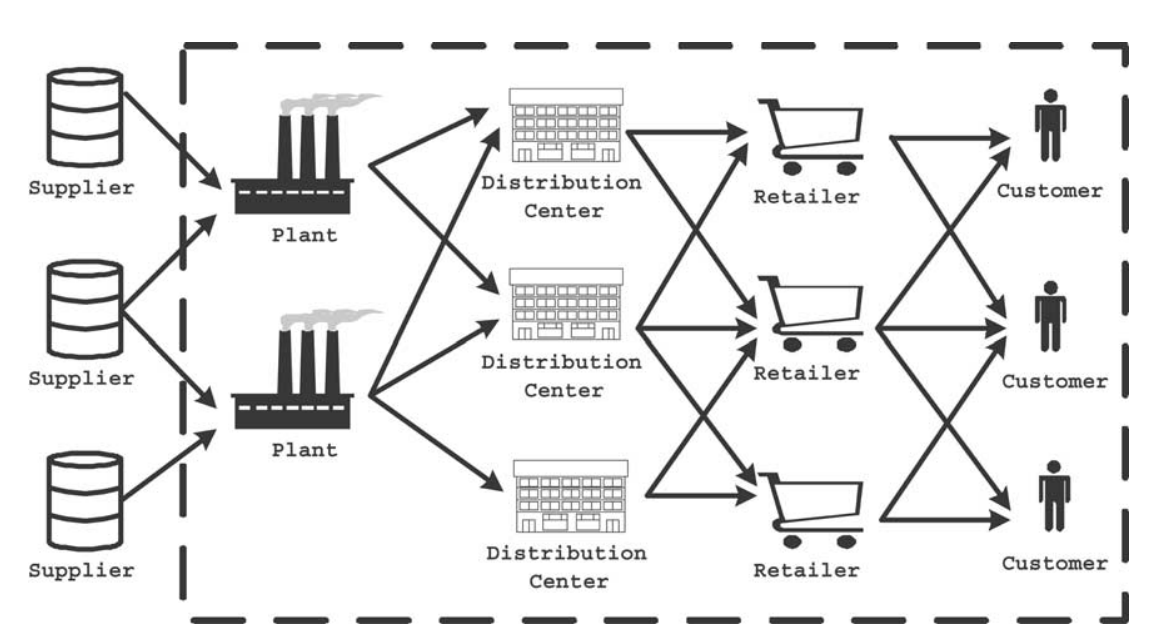

Fig. 1. Research region. 
the purchasing cost into manufacturing cost. The research region of this paper, therefore, is from manufacturer to customer, like the dash line region showed in Fig. 1. And the following assumptions have been made:

1. Products are independent to each other, related to marketing and sales price.

2. Each enterprise has its own safe inventory quantity to reduce the influence of uncertain product demand.

3. Several scenarios of product demands with known probabilities are forecasted over the entire scheduling periods.

4. The buyer's acceptability for product price can be quite different from the provider's.

The overall problem can thus be stated as follows:

\section{- Given:}

1. Manufacture data, such as batch manufacturing quantity of regular time and overtime, overtime number constraint, etc.

2. Transportation data, such as lead time, transport capacity, etc.

3. Inventory data, such as inventory capacity, safe inventory quantity, etc.

4. Each cost parameter, such as manufacturing, inventory, etc.

5. The buyers' and providers' acceptable levels for product prices.

6. Several scenarios of forecasted product demands with known probabilities.

- Determine:

1. Production plan of each plant.

2. Transportation plan of each DC.

3. Sales quantity and compromised product price of each participant.

4. Inventory level of each enterprise.

5. All kinds of cost.

- The target is to integrate the multi-echelon decisions simultaneously to:

1. Guarantee a fair profit distribution among all participants.

2. Elevate the customer service levels, the safe inventory levels, the product-prices satisfaction levels, and the robustness of all considered objectives to product demand uncertainties as much as possible.

\section{Uncertainties in the supply chain scheduling}

In the market, the participants of a supply chain not only face the uncertainties of product demands and raw material supplies but also faces the uncertainties of commodity prices and costs (Liu \& Sahinidis, 1997). The first concern in incorporating uncertainties into supply chain modeling and optimization is the determination of suitable representation of the uncertain parameters (Gupta \& Maranas, 2003). Three distinct methods are frequently mentioned for representing uncertainty (Liu \& Sahinidis, 1997; Gupta
\& Maranas, 2003): first, the distribution-based approach, where the normal distribution with specified mean and standard deviation is widely invoked for modeling uncertain demands and/or parameters; second, the fuzzy-based approach, therein the forecast parameters are considered as fuzzy numbers with accompanied membership functions; and third, the scenario-based approach, in which several discrete scenarios with associated probability levels are used to describe expected occurrence of particular outcomes. We will address issues of demand uncertainty and uncertain product prices in the following.

To simplify the subsequent mathematical calculations, we will adopt the discrete scenario-based approach for modeling uncertain product demands. For applying the discrete cases representation for modeling uncertain demands, several possible outcomes with known probabilities, $\mathrm{PPD}_{s}, s \in$ $\mathcal{S}$ where $\sum_{\forall s \in \mathcal{S}} \mathrm{PPD}_{s}=1$, should be determined at first. Then all variables will become scenario dependent, and the expected value of any variable will be the weighted average of those scenario-dependent values. That is, for any variable $v$, we have to solve for several scenario-dependent values, $v_{s}, s \in \mathcal{S}$, and the expected value of $v$ can be taken as $\sum_{\forall s \in \mathcal{S}} \mathrm{PPD}_{s} v_{s}$. In such a case the deterministic supply chain model can be easily extended to cope with uncertain demand conditions, as shown in the next section.

Due to the obvious negotiable and uncertain characteristics of products' prices at various selling sites in real businesses, the final product prices are usually result of compromised considerations. When contemplating the competitive positions hold between sellers and buyers in settling sales/purchase prices for a specific product, the preference of the price would be very different from each one's point of view. For example, the retailer would be fully satisfied if the selling price to customers is higher than an expected high value, say (USP) ${ }_{S}^{1}$; on the other hand, it would be totally unacceptable for a price less than a lower minimum (USP) ${ }_{S}^{0}$; and the degree-of-acceptability will increase in accordance with the increase of price between these two bounds. To describe such a transition from numerical price value to linguistic preference expression, it is quite suitable to set up a fuzzy set, $\mathcal{S P}$, with some kinds of monotonic increasing membership function, $\mu_{\mathcal{S P}}(\mathrm{USP})$ where $\mu_{\mathcal{S P}}\left(\mathrm{USP} \leq(\mathrm{USP})_{S}^{0}\right)=0$, $\mu_{\mathcal{S P}}\left(\mathrm{USP} \geq(\mathrm{USP})_{S}^{1}\right)=1$, and $\mu_{\mathcal{S P}}\left((\mathrm{USP})_{S}^{0}<\mathrm{USP}<\right.$ $\left.(\mathrm{USP})_{S}^{1}\right)=\mathbb{F}_{\text {inc }}\left(\mathrm{USP} ;(\mathrm{USP})_{S}^{0}\right.$, (USP) $\left.{ }_{S}^{1}\right) \in[0,1]$, to measure the seller's preference for product price. The buyer side, on the other hand, has its own fuzzy preference of purchase price, $\mathcal{B P}$, and corresponding monotonic decreasing membership function, $\mu_{\mathcal{B P}}$ (USP) where $\mu_{\mathcal{B} \mathcal{P}}\left(\mathrm{USP} \leq(\mathrm{USP}){ }_{B}^{1}\right)=$ $1, \mu_{\mathcal{B P}}\left(\mathrm{USP} \geq(\mathrm{USP})_{B}^{0}\right)=0$, and $\mu_{\mathcal{B P}}\left((\mathrm{USP}){ }_{B}^{1}<\mathrm{USP}<\right.$ $\left.(\mathrm{USP})_{B}^{0}\right)=\mathbb{F}_{\text {dec }}\left(\mathrm{USP} ;(\mathrm{USP}){ }_{B}^{1},(\mathrm{USP})_{B}^{0}\right) \in[0,1]$. The determination of membership functions are usually based on decision maker's subjectivity. It has been shown that use of linear membership functions can provide similar solution quality to that using more complicated nonlinear membership functions (Delgado, Herrera, \& Verdegay, 1993; Sakawa, 1993; Liu \& Sahinidis, 1997). Thus, we will assume 
(a)

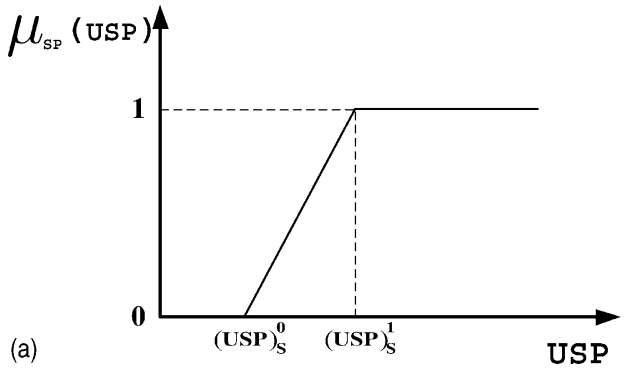

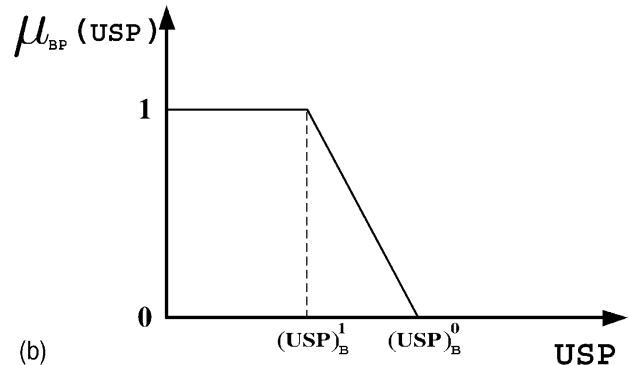

Fig. 2. Membership functions for seller's (a) and buyer's (b) fuzzy product prices.

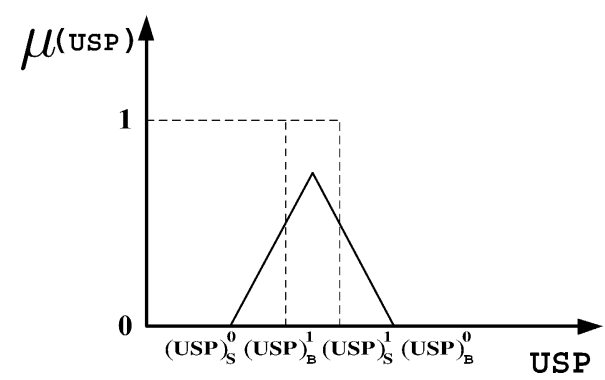

Fig. 3. Typical membership functions for fuzzy product prices when simultaneously considering the seller's and buyer's viewpoints.

constant rate of increased/decreased membership satisfaction and will adopt linear membership functions, as shown below and Fig. 2.

$$
\begin{aligned}
& \mu_{\mathcal{S P}}(\mathrm{USP}) \\
& = \begin{cases}0 & \text { for } \mathrm{USP} \leq(\mathrm{USP})_{S}^{0} \\
\frac{\mathrm{USP}-(\mathrm{USP})_{S}^{0}}{(\mathrm{USP})_{S}^{1}-(\mathrm{USP})_{S}^{0}} & \text { for }(\mathrm{USP})_{S}^{0} \leq \mathrm{USP} \leq(\mathrm{USP})_{S}^{1} \\
1 & \text { for USP } \geq(\mathrm{USP})_{S}^{1}\end{cases} \\
& \mu_{\mathcal{B} \mathcal{P}(\mathrm{USP})} \\
& = \begin{cases}1 & \text { for } \mathrm{USP} \leq(\mathrm{USP})_{B}^{1} \\
\frac{(\mathrm{USP})_{B}^{0}-\mathrm{USP}}{(\mathrm{USP})_{B}^{0}-(\mathrm{USP})_{B}^{1}} & \text { for }(\mathrm{USP})_{B}^{1} \leq \mathrm{USP} \leq(\mathrm{USP})_{B}^{0} \\
0 & \text { for } \mathrm{USP} \geq(\mathrm{USP})_{B}^{0}\end{cases}
\end{aligned}
$$

The final product price is determined by a compromising procedure taken place between these conflicting fuzzy preferences. Some typical final membership functions of the fuzzy product price-incorporating both the seller's and buyer's-are depicted in Fig. 3.

\section{Supply chain modeling with demand uncertainty}

A general supply chain that consists of three different levels of enterprises is considered here: the first level enterprise is the retailer from which the products are sold to customers; the second level enterprise is the distribution center (DC) or warehouse using different type of transport capacity to deliver products from plant side to retailer side; the third level enterprise is the plant or the manufacturer that batch-manufactures one product at one period. In the following, we develop an integrated multi-echelon supply chain model for optimal decisions (Chen et al., 2003). The scenario-based representation for uncertain product demands is considered in the modeling.

\subsection{Indices, sets, parameters, and variables}

The indices, sets and parameters, designed to model the supply chain network with product demand uncertainty are shown in the nomenclature. Therein, parameters are divided into two categories: the cost parameters, including inventory cost and transport cost; and other parameters describing the system information, such as inventory capacity, transport lead time, etc. Two kinds of scenario-dependent variables are used: the binary variables that act as policy decisions to use economies of scale for manufacturing or transportation, and continuous variables that include manufacturing quantities and product prices.

\subsection{Manufacture constraints}

Six constraints on manufacturing are set up for all products and plants over concerned periods.

$\sum_{\forall i \in \mathcal{I}} \beta_{p t s}^{i}=1$

$o_{p t s}^{i} \leq \alpha_{p t s}^{i} \leq \beta_{p t s}^{i}$

$\gamma_{p t s}^{i} \geq \beta_{p t s}^{i}-\beta_{p, t-1, s}^{i}$

$\sum_{\forall i \in \mathcal{I}} \sum_{\forall t \in \mathcal{T}} o_{p t s}^{i} \leq \mathrm{MTO}_{p}$

$\sum_{\forall i \in \mathcal{I}} \sum_{\forall n \in \mathcal{N}} o_{p, t-n+1, s}^{i} \leq N-1$

where $i \in \mathcal{I}, p \in \mathcal{P}, t \in \mathcal{T}, s \in \mathcal{S}$.

Eq. (2) denotes that the plant can be setup for manufacturing one product. Eq. (3) states that the plant is able to manu- 
facture a product in regular time only after the plant has been setup to produce it, and the plant is able to manufacture the product in overtime only when the regular time production is insufficient. In Eq. (4), $\gamma_{p t s}^{i}=1$ only if $\beta_{p t s}^{i}-\beta_{p, t-1, s}^{i}=1$, thus the plant can be changed to manufacture product $i$ at period $t$. Eq. (5) implies total number of overtime periods is less than the maximal allowable overtime periods, $\mathrm{MTO}_{p}$. And Eq. (6) says the number of continuous overtime periods should be less than a specified value $N$. Notably, these constraints are scenario dependent.

\subsection{Transportation constraints}

The transportation constraints at considered periods, $t \in$ $\mathcal{T}$, in different economic scales are given below.

$$
\begin{aligned}
& \mathrm{TCL}_{p d}^{k^{\prime}-1} Y_{p d t s}^{k^{\prime}}<\mathrm{TQ}_{p d t s}^{k^{\prime}} \leq \mathrm{TCL}_{p d}^{k^{\prime}} Y_{p d t s}^{k^{\prime}} \\
& \mathrm{TCL}_{d r}^{k-1} Y_{d r t s}^{k}<\mathrm{TQ}_{d r t s}^{k} \leq \mathrm{TCL}_{d r}^{k} Y_{d r t s}^{k} \\
& \sum_{\forall k^{\prime} \in \mathcal{K}^{\prime}} Y_{p d t s}^{k^{\prime}} \leq 1, \quad \sum_{\forall k \in \mathcal{K}} Y_{d r t s}^{k} \leq 1 \\
& \mathrm{TQ}_{p d t s}=\sum_{\forall k^{\prime} \in \mathcal{K}^{\prime}} \mathrm{TQ}_{p d t s}^{k^{\prime}}=\sum_{\forall i \in \mathcal{I}} \mathrm{SQ}_{p d t s}^{i} \\
& \mathrm{TQ}_{d r t s}=\sum_{\forall k \in \mathcal{K}} \mathrm{TQ}_{d r t s}^{k}=\sum_{\forall i \in \mathcal{I}} \mathrm{SQ}_{d r t s}^{i} \\
& \sum_{\forall p \in \mathcal{P}} \mathrm{TQ}_{p d t s} \leq \mathrm{MITC}_{d} \\
& \sum_{\forall r \in \mathcal{R}} \mathrm{TQ}_{d r t s} \leq \mathrm{MOTC}_{d}
\end{aligned}
$$

$\forall d \in \mathcal{D}, \quad p \in \mathcal{P}, \quad r \in \mathcal{R}, \quad k^{\prime} \in \mathcal{K}^{\prime}$,

$k \in \mathcal{K}, \quad t \in \mathcal{T}, \quad s \in \mathcal{S}$

Eqs. (7)-(9) imply that several transport-capacity levels with various unit transport costs can be used, as depicted in Fig. 4 for a three-level case, and at most one transport capacity can be chosen at each period. In Eqs. (10) and (11), the transport quantities of each product from plants to DCs or from DCs to retailers at each period are respectively translated into total transport quantities, and Eqs. (12) and (13) are constraints on these total transport quantities.

\subsection{Inventory constraints}

All relevant inventory constraints in all plants, DCs, and retailers can be summarized as follows:

$$
\begin{aligned}
& I_{r t s}^{i}=I_{r, t-1, s}^{i}+\sum_{\forall d \in \mathcal{D}} \mathrm{SQ}_{d r, t-\mathrm{TLT}_{d r}, s}^{i}-\sum_{\forall c \in \mathcal{C}} \mathrm{SQ}_{r c t s}^{i} \\
& I_{d t s}^{i}=I_{d, t-1, s}^{i}+\sum_{\forall p \in \mathcal{P}} \mathrm{SQ}_{p d, t-\mathrm{TLT}_{p d}, s}^{i}-\sum_{\forall r \in \mathcal{R}} \mathrm{SQ}_{d r t s}^{i}
\end{aligned}
$$

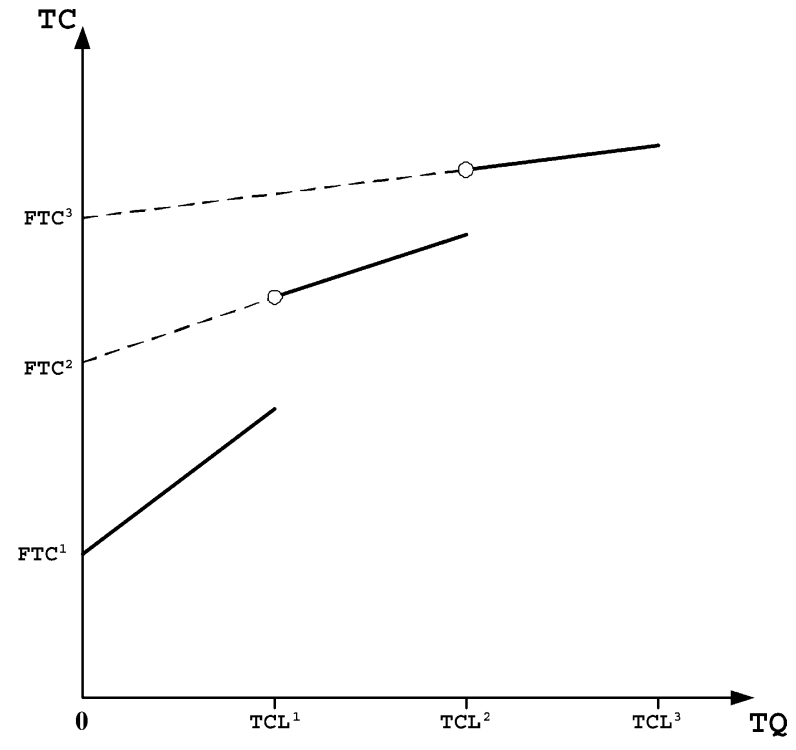

Fig. 4. Piecewise linear relation (solid lines) between transport cost (TC) and shipment quantity (TQ).

$$
\begin{aligned}
& I_{p t s}^{i}=I_{p, t-1, s}^{i}+\mathrm{FMQ}_{p}^{i} \alpha_{p, t-1, s}^{i} \\
& +\mathrm{OMQ}_{p}^{i} o_{p, t-1, s}^{i}-\sum_{\forall d \in \mathcal{D}} \mathrm{SQ}_{p d t s}^{i} \\
& B_{r t s}^{i}=B_{r, t-1, s}^{i}+\mathrm{FCD}_{r t s}^{i}-\sum_{\forall c \in \mathcal{C}} \mathrm{SQ}_{r c t s}^{i}, \quad B_{r T s}^{i}=0 \\
& \sum_{\forall i \in \mathcal{I}} I_{* t s}^{i} \leq \mathrm{MIC}_{*} \\
& \mathrm{SIQ}_{*}^{i}-I_{* t s}^{i} \leq D_{* t s}^{i} \\
& I_{* t s}^{i}, \mathrm{SQ}_{* t s}^{i}, B_{* t s}^{i}, D_{* t s}^{i} \geq 0
\end{aligned}
$$

where $* \in\{p, d, r\}, p \in \mathcal{P}, d \in \mathcal{D}, r \in \mathcal{R}, i \in \mathcal{I}, t \in \mathcal{T}$, $s \in \mathcal{S}$.

Here, Eq. (14) states that inventory level of one product of a retailer at each period equals the amounts at previous period plus the amounts received from all DCs and less the amounts sold to customers. Similar constraints apply for DCs and plants, as shown in Eqs. (15) and (16). Eqs. (14) and (15) also consider delayed transport quantity caused by transport lead time. Eq. (17) means that backlog level of one product equals the amounts at previous period and added to the amounts of forecasting customer demand, less the amounts sold to customer; and the backlog at the last period should be zero for fulfilling expected customer demand. Eq. (18) says that the amounts of all products can not exceed the maximal inventory capacity. By using safe inventory quantity constraints, we can make the short safe inventory level of a product to be zero if inventory level is greater than safe inventory quantity, or to be the difference of safe inventory quantity and inventory level if inventory level is smaller than safe inventory quantity, as shown in Eq. (19). 


\subsection{Costs and revenues}

The plants' manufacturing costs, purchasing costs for DCs, retailers and customers, all inventory and handling costs, and the transportation costs are listed in the following for all $p \in \mathcal{P}, d \in \mathcal{D}, r \in \mathcal{R}, t \in \mathcal{T}$, and $s \in \mathcal{S}$.

$$
\begin{aligned}
\mathrm{TMC}_{p t s}= & \sum_{\forall i \in \mathcal{I}} \mathrm{FMC}_{p}^{i} \gamma_{p t s}^{i}+\mathrm{FIC}_{p}^{i}\left(\beta_{p t s}^{i}\right. \\
& +\mathrm{UMC}_{p}^{i} \mathrm{FMQ}_{p}^{i} \alpha_{p t s}^{i}+\mathrm{OMC}_{p} \\
\mathrm{TPC}_{d t s}= & \sum_{\forall p \in \mathcal{P} \forall i \in \mathcal{I}} \sum_{\mathrm{TPC}_{r t s}=} \sum_{\forall d \in \mathcal{D}} \sum_{\forall i \in \mathcal{I}} \mathrm{USP}_{d r s}^{i} \mathrm{SQ}_{d r t s}^{i} \mathrm{SQ}_{p d t s}^{i}, \\
\mathrm{TIC}_{* t s}= & \sum_{\forall i \in \mathcal{I}} \mathrm{UIC}_{*}^{i} I_{* t s}^{i} \quad * \in\{p, d, r\}
\end{aligned}
$$

$\mathrm{THC}_{p t s}$

$$
\begin{aligned}
= & \sum_{\forall i \in \mathcal{I}} \mathrm{UHC}_{p}^{i} \\
& \times\left(\mathrm{FMQ}_{p}^{i} \alpha_{p, t-1, s}^{i}+\mathrm{OMQ}_{p}^{i} o_{p, t-1, s}^{i}+\sum_{\forall d \in \mathcal{D}} \mathrm{SQ}_{p d t s}^{i}\right)
\end{aligned}
$$

$$
\mathrm{THC}_{d t s}=\sum_{\forall i \in \mathcal{I}} \mathrm{UHC}_{d}^{i}\left(\sum_{\forall p \in \mathcal{P}} \mathrm{SQ}_{p d, t-\mathrm{TLT}_{p d}, s}^{i}+\sum_{\forall r \in \mathcal{R}} \mathrm{SQ}_{d r t s}^{i}\right)
$$

$\mathrm{THC}_{r t s}=\sum_{\forall i \in \mathcal{I}} \mathrm{UHC}_{r}^{i}\left(\sum_{\forall d \in \mathcal{D}} \mathrm{SQ}_{d r, t-\mathrm{TLT}_{d r}, s}^{i}+\sum_{\forall c \in \mathcal{C}} \mathrm{SQ}_{r c t s}^{i}\right)$

$\mathrm{TTC}_{p t s}=\sum_{\forall k^{\prime} \in \mathcal{K}^{\prime}} \sum_{\forall p \in \mathcal{P}}\left(\mathrm{FTC}_{p d}^{k^{\prime}} Y_{p d t s}^{k^{\prime}}+\mathrm{UTC}_{p d}^{k^{\prime}} \mathrm{TQ}_{p d t s}^{k^{\prime}}\right)$

$\mathrm{TTC}_{d t s}=\sum_{\forall k \in \mathcal{K}} \sum_{\forall r \in \mathcal{R}}\left(\mathrm{FTC}_{d r}^{k} Y_{d r t s}^{k}+\mathrm{UTC}_{d r}^{k} \mathrm{TQ}_{d r t s}^{k}\right)$

$\mathrm{PSP}_{p t s}=\sum_{\forall d \in \mathcal{D}} \sum_{\forall i \in \mathcal{I}} \mathrm{USP}_{p d s}^{i} \mathrm{SQ}_{p d t s}^{i}$,

$\operatorname{PSP}_{d t s}=\sum_{\forall r \in \mathcal{R}} \sum_{\forall i \in \mathcal{I}} \mathrm{USP}_{d r s}^{i} \mathrm{SQ}_{d r t s}^{i}$,

$\mathrm{PSP}_{r t s}=\sum_{\forall c \in \mathcal{C}} \sum_{\forall i \in \mathcal{I}} \mathrm{USP}_{r c s}^{i} \mathrm{SQ}_{r c t s}^{i}$

In Eq. (21), the manufacturing cost is a composite obtained by fixed manufacture and idle cost plus regular and overtime manufacturing costs. Here, the $\gamma_{p t s}^{i}$ value (measured if we are going to change production plan to produce product $i$ ) will be either zero (if $\beta_{p t s}^{i}-\beta_{p, t-1, s}^{i}=0$, continuing to produce or not to produce product $i$ ) or 1 (if $\beta_{p t s}^{i}-\beta_{p, t-1, s}^{i}=$ 1 , changeover to start producing product $i$ ). Eq. (22) gives the purchasing costs for DCs and retailers; Eq. (23) is the inventory cost, and Eqs. (24) and (26) are handling costs for plants, DCs, and retailers, respectively; Eqs. (27) and (28) are transport costs for plant and DC, respectively. Here, the transport cost is a composite of transport level-dependent fixed cost and a transport quantity-dependent carrying cost. This would cause a discontinuous piecewise linear transport cost, as illustrated in Fig. 4 with skipped subscripts. Notably, the discontinuities in the transport cost make the model more general than the continuous one proposed by Tsiakis et al. (2001). Finally, Eq. (29) is product sales for all plants, DCs, and retailers.

\subsection{Multiple objectives}

The conflict objectives such as each participant's profit, the average customer service level, and the average safe inventory level are considered simultaneously, as stated in the following.

Objective 1: to simultaneously maximize participants' expected profits for $p \in \mathcal{P}, d \in \mathcal{D}$, and $r \in \mathcal{R}$.

Instead of directly maximizing the overall profit of the integrated supply chain network, we intend to fairly distribute the profit to all members within scheduling periods. The profits of all participants to be maximized are considered separately, where the profit at period $t$ is equal to the product sales less all kinds of costs.

$$
Z_{*}=\sum_{\forall s \in \mathcal{S}} \operatorname{PPD}_{s} Z_{* s}, \quad * \in\{p, d, r\}
$$

where

$$
\begin{aligned}
& Z_{p s}= \sum_{\forall t \in \mathcal{T}}\left(\mathrm{PSP}_{p t s}-\mathrm{TMC}_{p t s}-\mathrm{TTC}_{p t s}\right. \\
&\left.-\mathrm{TIC}_{p t s}-\mathrm{THC}_{p t s}\right) \quad \forall p \in \mathcal{P}, s \in \mathcal{S} \\
& Z_{d s}= \sum_{\forall t \in \mathcal{T}}\left(\mathrm{PSP}_{d t s}-\mathrm{TPC}_{d t s}-\mathrm{TTC}_{d t s}\right. \\
&\left.-\mathrm{TIC}_{d t s}-\mathrm{THC}_{d t s}\right) \quad \forall d \in \mathcal{D}, s \in \mathcal{S} \\
& Z_{r s}= \sum_{\forall t \in \mathcal{T}}\left(\mathrm{PSP}_{r t s}-\mathrm{TPC}_{r t s}-\mathrm{THC}_{r t s}-\mathrm{TIC}_{r t s}\right) \\
& \quad \forall r \in \mathcal{R}, s \in \mathcal{S} .
\end{aligned}
$$

Objective 2: to maximize average safe inventory levels for $p \in \mathcal{P}, d \in \mathcal{D}, r \in \mathcal{R}$.

The safe inventory level of retailer $r$ at period $t$ for scenario $s$ is defined as the expected average percentage of 1 less the ratio of short safe inventory level of product $i$ of retailer $r$ at period $t, D_{r t s}^{i}$, over the safe inventory 
quantity of product $i$ of retailer $r, \mathrm{SIQ}_{r}^{i}$, for all products. Similar definitions are also applied to plants and DCs. All participants' safe inventory levels are concerned as objectives for simultaneous optimization.

$\mathrm{SIL}_{*}=\sum_{\forall s \in \mathcal{S}} \operatorname{PPD}_{s} \mathrm{SIL}_{* s}, \quad * \in\{p, d, r\}$

where

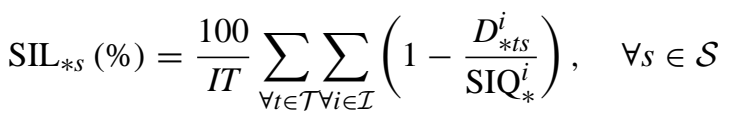

Objective 3: to maximize average customer service levels for $r \in \mathcal{R}$.

Under the condition of taking all products into consideration, the customer service level of retailer $r$ at period $t$ is defined as the expected average percentage ratio of actual sales quantity of product $i$ from retailer $r$ to customers at period $t, \sum_{\forall c \in \mathcal{C}} \mathrm{SQ}_{r c t s}^{i}$, over the expected total demand quantity. The expected total demand quantity is the sum of backlog level of product $i$ for retailer $r$ at the end of period $t-1, B_{r, t-1, s}^{i}$, and forecasting customer demand of product $i$ to retailer $r$ at period $t, \sum_{\forall c \in \mathcal{C}} \mathrm{FCD}_{r c t s}^{i}$.

$\mathrm{CSL}_{r}=\sum_{\forall s \in \mathcal{S}} \operatorname{PPD}_{s} \mathrm{CSL}_{r s}, \quad \forall r \in \mathcal{R}$

where

$$
\begin{aligned}
\operatorname{CSL}_{r s}(\%)= & \frac{100}{I T} \sum_{\forall t \in \mathcal{T}} \sum_{\forall i \in \mathcal{I}} \\
& \times\left(\frac{\sum_{\forall c \in \mathcal{C}} \mathrm{SQ}_{r c t s}^{i}}{B_{r, t-1, s}^{i}+\sum_{\forall c \in \mathcal{C}} \mathrm{FCD}_{r c t s}^{i}}\right), \quad \forall s \in \mathcal{S} .
\end{aligned}
$$

Objective 4: to maximize robustness of selected objectives to demand uncertainties.

It has been mentioned that all variables are scenarios dependent when the explicit scenario-based approach is applied to the uncertain product demand. However, the profit realization might be unacceptably low for certain scenarios with especially low probabilities (Suh \& Lee, 2001). It is thus significant to reduce the variability of above-mentioned objective values for any realization of scenarios. An important issue in enforcing robustness to uncertainties is the choice of variability metric (Ahmed \& Sahinidis, 1998). For those objectives to be maximized as mentioned previously, the decision-maker usually does not care if the objective value is greater than the expected one. We thus propose the lower partial mean as the measure of robustness, where only objective values less than the expectation are penalized and are weighted by probabilities of related scenarios.

$$
\mathrm{RI}_{m^{\prime}}=\sum_{\forall s \in \mathcal{S}} \operatorname{PPD}_{s} \min \left\{0, J_{m^{\prime} s}-J_{m^{\prime}}\right\}, \quad \forall m^{\prime} \in \mathcal{M}^{\prime}
$$

Here, $\mathcal{M}^{\prime}$ is the index set of objectives in Eqs. (30)-(32) with dimension $\left[\mathcal{M}^{\prime}\right]=M^{\prime}=2 P+2 D+3 R$.

In summary, the feasible searching space, $\boldsymbol{\Omega}$, are composite of all constraints mentioned above. The multiple objectives $J_{m}(\boldsymbol{x}), m \in \mathcal{M}$, where $\mathcal{M}$ is the index set of all objectives including the robustness indices with $[\mathcal{M}]=M=2 M^{\prime}$, and the decision vector $\boldsymbol{x}$ are shown below. Notably, further to the scenario-dependent product prices, the decision vector $\boldsymbol{x}$ includes all production, transportation, sales quantities and inventory levels during considered periods.

$$
\begin{aligned}
\max _{\boldsymbol{x} \in \boldsymbol{\Omega}} & \left(J_{1}(\boldsymbol{x}), \ldots, J_{M}(\boldsymbol{x})\right) \\
= & \left(Z_{p}, Z_{d}, Z_{r} ; \mathrm{SIL}_{p}, \mathrm{SIL}_{d}, \mathrm{SIL}_{r} ; \mathrm{CSL}_{r} ; \mathrm{RI}_{m^{\prime}} ;\right. \\
& \left.\forall p \in \mathcal{P}, d \in \mathcal{D}, r \in \mathcal{R}, m^{\prime} \in \mathcal{M}^{\prime}\right) \\
\boldsymbol{x}= & \left\{\alpha_{p t s}^{i}, \beta_{p t s}^{i}, \gamma_{p t s}^{i}, o_{p t s}^{i} ; \mathrm{SQ}_{* t s}^{i} ; \mathrm{USP}_{* s}^{i} ; \mathrm{TQ}_{p d t s}^{k^{\prime}}, \mathrm{TQ}_{d r t s}^{k},\right. \\
& Y_{p d t s}^{k^{\prime}}, Y_{d r t s}^{k} ; I_{p t s}^{i}, I_{d t s}^{i}, I_{r t s}^{i} ; B_{r t s}^{i} ; D_{p t s}^{i}, D_{d t s}^{i}, D_{r t s}^{i} ; \\
& * \in\{p d, d r, r c\} ; i \in \mathcal{I}, p \in \mathcal{P}, d \in \mathcal{D}, r \in \mathcal{R}, \\
& \left.c \in \mathcal{C}, k^{\prime} \in \mathcal{K}^{\prime}, k \in \mathcal{K}, t \in \mathcal{T}, s \in \mathcal{S}\right\}
\end{aligned}
$$

\section{Supply chain optimization with uncertain demands and prices}

By considering the uncertain property of human thinking, it is quite intuitive to assume that the DM has a fuzzy goal, $\mathcal{J}_{m}$, to describe the maximizing objective $J_{m}$ with an acceptable interval $\left[J_{m}^{0}, J_{m}^{1}\right]$. It would be quite satisfactory as the objective value is greater than $J_{m}^{1}$, and unacceptable as the profit is less than $J_{m}^{0}$, the minimum acceptable objective value such that the company would like to enter to negotiation for a fair deal in the multi-enterprise network. A strictly monotonic increasing membership function, $\mu_{\mathcal{J}_{m}}\left(J_{m}(\boldsymbol{x})\right) \in$ $[0,1]$, can be used to characterize such a transition from numerical objective value $J_{m}(\boldsymbol{x})$ to degree-of-satisfaction for $\mathcal{J}_{m}$. Without loss of generality, we will adopt the linear membership function since it has been proved in providing qualified solutions for many applications (Liu \& Sahinidis, 1997).

$$
\begin{aligned}
& \mu_{\mathcal{J}_{m}}\left(J_{m}(\boldsymbol{x})\right) \\
& = \begin{cases}1 ; & \text { for } J_{m}(\boldsymbol{x}) \geq J_{m}^{1} \\
\frac{J_{m}(\boldsymbol{x})-J_{m}^{0}}{J_{m}^{1}-J_{m}^{0}} ; & \text { for } J_{m}^{0} \leq J_{m}(\boldsymbol{x}) \leq J_{m}^{1} \quad \forall m \in \mathcal{M} \\
0 ; & \text { for } J_{m}^{0} \geq J_{m}(\boldsymbol{x})\end{cases}
\end{aligned}
$$

Here, $\boldsymbol{x}$ denotes the argument vector as shown in Eq. (35). The effective range of the membership function, $\left[J_{m}^{0}, J_{m}^{1}\right]$, can sometimes be subjectively defined by company's decision makers. Some procedures can also follow for providing reasonable limiting values for the objective. For those objectives such as profits and inventory levels and customer 
service levels, $J_{m^{\prime}}, m^{\prime} \in \mathcal{M}^{\prime}$, one can use the most optimistic expectation as the upper limit, $\bar{J}_{m^{\prime}}^{1}=J_{m^{\prime}}\left(\boldsymbol{x}_{m^{\prime}}^{*}\right)$, where $\boldsymbol{x}_{m^{\prime}}^{*}$ is the optimal solution of the single objective maximizing problem, $\max _{\boldsymbol{x} \in \boldsymbol{\Omega}} J_{m^{\prime}}(\boldsymbol{x})$. And choose the most pessimistic expectation, $\underline{J}_{m}^{0}$, as the lower limiting value (Zimmermann, 1978; Sakawa, 1993), where

$\underline{J}_{m^{\prime}}^{0}=\min \left\{J_{m^{\prime}}\left(\boldsymbol{x}_{i}^{*}\right), i \in \mathcal{M}^{\prime}\right\}, \quad \forall m^{\prime} \in \mathcal{M}^{\prime}$

As for objectives measuring robustness to uncertainties, the reasonable upper limit is zero (i.e., absolute robustness), $\bar{J}_{M^{\prime}+m^{\prime}}^{1}=0$, and the lower limiting value can be found similar to Eq. (37).

$\underline{J}_{M^{\prime}+m^{\prime}}^{0}=\min \left\{J_{M^{\prime}+m^{\prime}}\left(\boldsymbol{x}_{i}^{*}\right), i \in \mathcal{M}^{\prime}\right\}, \quad \forall m^{\prime} \in \mathcal{M}^{\prime}$

One can thus subjectively determine the effective range for membership functions with the restriction of $J_{m}^{0} \leq J_{m}^{0}<$ $J_{m}^{1} \leq \bar{J}_{m}^{1}$. The original multi-objective optimization problem is now equivalent to look for a suitable decision vector that can provide the maximal degree-of-satisfaction for the aggregated fuzzy objectives, $\mathcal{J}_{1} \cap \ldots \cap \mathcal{J}_{M}$. When simultaneously considering the incompatible fuzzy preference on product prices from sellers and buyers viewpoints, the final fuzzy decision, $\mathcal{F D}$, can be interpreted as the fuzzy intersection between all fuzzy objectives and fuzzy product prices.

$$
\begin{aligned}
& \mathcal{F D}=\mathcal{J}_{m} \cap \mathcal{S P}_{p d}^{i} \cap \mathcal{S P}_{d r}^{i} \cap \mathcal{S P}_{r c}^{i} \cap \mathcal{B} \mathcal{P}_{p d}^{i} \cap \mathcal{B P}_{d r}^{i} \cap \mathcal{B} \mathcal{P}_{r c}^{i} \\
& \forall m \in \mathcal{M}, i \in \mathcal{I}, p \in \mathcal{P}, d \in \mathcal{D}, r \in \mathcal{R}, c \in \mathcal{C}
\end{aligned}
$$

Noted that the expected product prices, $\mathrm{USP}_{*}^{i}=\sum_{\forall s \in \mathcal{S}}$ $\mathrm{PPD}_{s} \mathrm{USP}_{* s}^{i}, * \in\{p d, d r, r c\}$, should be used in evaluating the degree-of-acceptability of various fuzzy preferences. The final overall satisfactory level, $\mu_{\mathcal{F D}}(\boldsymbol{x})$, can be determined by aggregating the degree-of-satisfaction for all objectives, $\mu_{\mathcal{J}_{m}}\left(J_{m}(\boldsymbol{x})\right)$, and sellers' and buyers' preference on product prices, $\mu_{\mathcal{S P}}\left(\mathrm{USP}_{*}^{i}\right)$ and $\mu_{\mathcal{B} \mathcal{P}_{*}^{i}}\left(\mathrm{USP}_{*}^{i}\right)$, via specific $t$-norm, $\mathbb{T}$.

$$
\begin{aligned}
\mu_{\mathcal{F D}}(\boldsymbol{x})= & \mathbb{T}\left(\mu_{\mathcal{J}_{m}} ; \mu_{\mathcal{S P}}^{i}{ }_{p d}, \mu_{\mathcal{S P}}^{i}{ }_{d r}, \mu_{\mathcal{S P}}^{i} ;\right. \\
& \left.\mu_{\mathcal{B P}_{p d}^{i}}, \mu_{\mathcal{B} \mathcal{P}_{d r}^{i}}, \mu_{\mathcal{B} \mathcal{P}_{r c}^{i}} \mid \forall m, i, p, d, r, c\right)
\end{aligned}
$$

The best solution $\boldsymbol{x}^{*}$ with the maximal firing level, $\mu_{\mathcal{F D}}\left(\boldsymbol{x}^{*}\right)$, should be selected.

$\mu_{\mathcal{F D}}\left(\boldsymbol{x}^{*}\right)=\max _{\boldsymbol{x} \in \boldsymbol{\Omega}} \mu_{\mathcal{F D}}(\boldsymbol{x})$

Several $t$-norms can be chosen for $\mathbb{T}$, therein two most popular selections are shown below (Klir \& Yuan, 1995).

$$
\begin{aligned}
& \mathbb{T}\left(\mu_{\mathcal{J}_{m}} ; \mu_{\mathcal{S} \mathcal{P}_{p d}^{i}}, \mu_{\mathcal{S} \mathcal{P}_{d r}^{i}}, \mu_{\mathcal{S P}_{r c}^{i}} ; \mu_{\mathcal{B} \mathcal{P}_{p d}^{i}}, \mu_{\mathcal{B P}_{d r}^{i}}, \mu_{\mathcal{B P}_{r c}^{i}}\right)
\end{aligned}
$$

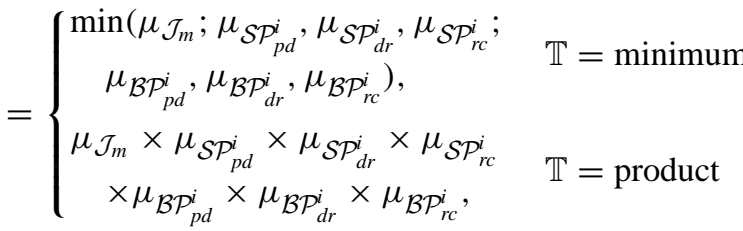

The minimum operator concerns the worst situation only, and the product operator results in a Nash-type objective. Maximizing the single worst scenario may end in a non-compensatory solution, and maximizing the Nash-type objective can guarantee a compensatory solution ( $\mathrm{Li} \& \mathrm{Lee}$, 1993). But the drawback is that product operator may cause an unbalanced solution between all fuzzy terms by the inherent character of product. We thus propose a two-phase method to combine advantages of these two operators, as summarized in the following (Chen et al., 2003).

Step 1. Determining membership function for each fuzzy objective based on expected upper/lower bounds for the objective value, as shown in Eq. (36), where

$\underline{J}_{m}^{0} \leq J_{m}^{0} \leq J_{m}^{1} \leq \bar{J}_{m}^{1}, \quad \forall m \in \mathcal{M}$.

Step 2 (Phase I). Considering all fuzzy objectives and fuzzy product prices and using the minimum operator, maximizing the degree of satisfaction for the worst situation.

$$
\begin{aligned}
\max _{\boldsymbol{x} \in \boldsymbol{\Omega}} \mu_{\mathcal{F D}}= & \max _{\boldsymbol{x} \in \boldsymbol{\Omega}} \min \left(\mu_{\mathcal{J}_{m}} ; \mu_{\mathcal{S P}_{p d}^{i}}, \mu_{\mathcal{S P}_{d r}^{i}}, \mu_{\mathcal{S P}_{r c}^{i}}\right. \\
& \left.\mu_{\mathcal{B} P_{p d}^{i}}, \mu_{\mathcal{B P}_{d r}^{i}}, \mu_{\mathcal{B P}_{r c}^{i}}\right) \equiv \mu_{\min }
\end{aligned}
$$

Step 3 (Phase II). Applying the product operator, maximiz-

\begin{tabular}{|c|c|c|c|c|c|c|c|c|c|}
\hline \multirow[t]{2}{*}{$i$} & \multirow[t]{2}{*}{$r$} & \multirow[t]{2}{*}{$t$} & \multicolumn{7}{|c|}{$\mathrm{FCD}_{r t s}^{i}$} \\
\hline & & & $0.15^{\mathrm{a}}$ & $0.2^{\mathrm{a}}$ & $0.12^{\mathrm{a}}$ & $0.24^{\mathrm{a}}$ & $0.09^{\mathrm{a}}$ & $0.13^{\mathrm{a}}$ & $0.07^{\mathrm{a}}$ \\
\hline 1 & 1 & 1 & 160 & 160 & 160 & 160 & 160 & 160 & 160 \\
\hline 1 & 1 & 2 & 170 & 170 & 170 & 160 & 150 & 150 & 150 \\
\hline 1 & 1 & 3 & 180 & 180 & 180 & 160 & 140 & 140 & 140 \\
\hline 1 & 1 & 4 & 190 & 180 & 170 & 160 & 150 & 140 & 130 \\
\hline 1 & 1 & 5 & 200 & 180 & 160 & 160 & 160 & 140 & 120 \\
\hline 1 & 2 & 1 & 180 & 180 & 180 & 180 & 180 & 180 & 180 \\
\hline 1 & 2 & 2 & 200 & 200 & 180 & 200 & 160 & 160 & 160 \\
\hline 1 & 2 & 3 & 220 & 220 & 180 & 220 & 140 & 140 & 140 \\
\hline 1 & 2 & 4 & 220 & 240 & 180 & 200 & 140 & 120 & 160 \\
\hline 1 & 2 & 5 & 220 & 260 & 180 & 180 & 140 & 100 & 180 \\
\hline 2 & 1 & 1 & 240 & 240 & 240 & 240 & 240 & 240 & 240 \\
\hline 2 & 1 & 2 & 240 & 270 & 210 & 210 & 270 & 270 & 210 \\
\hline 2 & 1 & 3 & 240 & 300 & 180 & 180 & 300 & 300 & 180 \\
\hline 2 & 1 & 4 & 240 & 270 & 210 & 150 & 330 & 300 & 180 \\
\hline 2 & 1 & 5 & 240 & 240 & 240 & 120 & 360 & 300 & 180 \\
\hline 2 & 2 & 1 & 280 & 280 & 280 & 280 & 280 & 280 & 280 \\
\hline 2 & 2 & 2 & 320 & 240 & 320 & 320 & 240 & 240 & 280 \\
\hline 2 & 2 & 3 & 360 & 200 & 360 & 360 & 200 & 200 & 280 \\
\hline 2 & 2 & 4 & 320 & 200 & 400 & 360 & 160 & 240 & 280 \\
\hline 2 & 2 & 5 & 280 & 200 & 400 & 360 & 120 & 280 & 280 \\
\hline
\end{tabular}
ing the overall Nash-type satisfactory level with guaranteed minimal fulfillment for all fuzzy objectives and sales preferences.

Table 1

Scenarios ( $s=1,2,3,4,5,6$ and 7$)$ of forecasting product demands and probabilities of illustrative example 


$$
\begin{aligned}
\max _{\boldsymbol{x} \in \boldsymbol{\Omega}^{+}} \mu_{\mathcal{F D}}(\boldsymbol{x})= & \max _{\boldsymbol{x} \in \boldsymbol{\Omega}^{+}}\left(\mu_{\mathcal{J}_{m}} \times \mu_{\mathcal{S P}}^{i}{ }_{p d}^{i} \times \mu_{\mathcal{S P}}^{i} \times \mu_{\mathcal{S P}_{r c}^{i}}\right. \\
& \left.\times \mu_{\mathcal{B} \mathcal{P}_{p d}^{i}} \times \mu_{\mathcal{B} \mathcal{P}_{d r}^{i}} \times \mu_{\mathcal{B P}_{r c}^{i}}\right)
\end{aligned}
$$

where

$$
\begin{aligned}
\Omega^{+}= & \Omega \cap\left\{\mu_{\mathcal{J}_{m}}, \mu_{\mathcal{S P}_{*}^{i}}, \mu_{\mathcal{B P}_{*}^{i}} \geq \mu_{\min } \mid * \in\{p d, d r, r c\} ;\right. \\
& \forall m, i, p, d, r, c\}
\end{aligned}
$$

\section{Numerical example}

Considering a small-scale but typical supply chain consists of one plant, two DCs, two retailers, and two products. The first DC, a smaller scale but faster delivery service distributor, can rapidly respond to the suddenly increasing customer demand to keep retailer's customer service level, but this also implies a higher operational cost; the second one, a large scale but slower delivery service distributor, on the other hand, can use the economies of scale to transport goods at lower operational cost, but the prompt delivery is not available, however. We assume one period of transport lead time between each level for the second DC. So, the distribution channel between plants to retailers is complementary with the faster, smaller shipment and slower, larger

\begin{tabular}{|c|c|c|c|c|c|c|c|c|c|c|}
\hline \multicolumn{7}{|c|}{ Unit Inventory Cost Unit Transport Cost } & \multicolumn{4}{|c|}{ Fix Transport Cost } \\
\hline & \multicolumn{2}{|c|}{$i p d r$} & \multirow{2}{*}{\multicolumn{2}{|c|}{$\begin{array}{r}\$ \\
50\end{array}$}} & \multicolumn{2}{|c|}{$k p d r$} & $\$$ & \multicolumn{2}{|c|}{$k p d r$} & \multirow{2}{*}{$\begin{array}{r}\$ \\
1800\end{array}$} \\
\hline & 1 & 1 & & & 1 & 11 & 60 & 1 & 11 & \\
\hline \multirow[t]{4}{*}{$\mathrm{UIC}_{r}^{i}$} & 1 & 2 & 40 & & 2 & 11 & 45 & 2 & 11 & 2700 \\
\hline & 2 & 1 & 30 & & 3 & 11 & 33 & 3 & 11 & 3200 \\
\hline & 2 & 2 & 24 & & 1 & 12 & 50 & 1 & 12 & 1500 \\
\hline & 1 & 1 & 45 & & 2 & 12 & 37 & 2 & 12 & 2200 \\
\hline \multirow[t]{3}{*}{$\mathrm{UIC}_{d}^{i}$} & 1 & 2 & 20 & $\mathrm{UTC}_{d r}^{k}$ & 3 & 12 & $27 \mathrm{FTC}_{d r}^{k}$ & 3 & 12 & 2600 \\
\hline & 2 & 1 & 27 & & 1 & 21 & 25 & 1 & 21 & 3000 \\
\hline & 2 & 2 & 12 & & 2 & 21 & 18 & 2 & 21 & 5250 \\
\hline \multirow[t]{2}{*}{$\mathrm{UIC}_{p}^{i}$} & 11 & & 15 & & 3 & 21 & 14 & 3 & 21 & 6750 \\
\hline & 21 & & 9 & & 1 & 22 & 30 & 1 & 22 & 2000 \\
\hline \multicolumn{5}{|c|}{$\overline{\text { Unit Handling Cost }}$} & & 22 & 22 & 2 & 22 & 3500 \\
\hline & 1 & 1 & 15 & & 3 & 22 & 16 & 3 & 22 & 4500 \\
\hline \multirow[t]{4}{*}{$\mathrm{UHC}_{r}^{i}$} & 1 & 2 & 12 & & 11 & & 40 & 11 & & 2000 \\
\hline & 2 & 1 & 9 & & 21 & & 30 & 21 & & 3000 \\
\hline & 2 & 2 & & $\mathrm{UTC}_{p d}^{k^{\prime}}$ & 31 & & $20 \mathrm{FTC}_{p d}^{k^{\prime}}$ & 31 & & 3500 \\
\hline & 1 & 1 & 12 & & 11 & & 20 & 11 & & 3200 \\
\hline \multirow{3}{*}{$\mathrm{UHC}_{d}^{i}$} & 1 & 2 & 6 & & 21 & & 15 & 21 & & 5600 \\
\hline & 2 & 1 & 7 & & 31 & & 10 & 31 & & 7200 \\
\hline & 2 & 2 & 4 & Unit 1 & Manı & if. $\mathrm{Cc}$ & Fix M & Ianu & f/Idle & Cost \\
\hline \multirow[t]{5}{*}{$\mathrm{UHC}_{p}^{i}$} & 11 & & 5 & & $i p$ & & $\$$ & $i p$ & & $\$$ \\
\hline & 21 & & & $\mathrm{UMC}_{p}^{i}$ & 11 & & $60 \mathrm{FMC}_{p}^{i}$ & 11 & & 48000 \\
\hline & & & & & 21 & & 30 & 21 & & 36000 \\
\hline & & & & $\mathrm{OMC}_{p}^{i}$ & 11 & & $90 \mathrm{FIC}_{p}^{i}$ & 11 & & 4800 \\
\hline & & & & & 21 & & 45 & 21 & & 3600 \\
\hline
\end{tabular}
shipment. And in order to simplify the problem, we neglect

Table 2

Cost parameters of illustrative example

\begin{tabular}{|c|c|c|c|c|c|c|c|c|}
\hline \multirow[t]{2}{*}{$\bullet$} & \multirow[t]{2}{*}{$i$} & \multirow[t]{2}{*}{$p$} & \multirow[t]{2}{*}{$d$} & \multirow[t]{2}{*}{$r$} & \multicolumn{2}{|l|}{ Seller } & \multicolumn{2}{|l|}{ Buyer } \\
\hline & & & & & $(\bullet)_{S}^{0}$ & $(\bullet)_{S}^{1}$ & $(\bullet)_{B}^{1}$ & $(\bullet)_{B}^{0}$ \\
\hline \multirow[t]{8}{*}{$\operatorname{USP}_{d r}^{i}$} & 1 & & 1 & 1 & 1350 & 1450 & 1400 & 1500 \\
\hline & 1 & & 1 & 2 & 1400 & 1500 & 1450 & 1550 \\
\hline & 1 & & 2 & 1 & 1250 & 1350 & 1300 & 1400 \\
\hline & 1 & & 2 & 2 & 1200 & 1300 & 1250 & 1350 \\
\hline & 2 & & 1 & 1 & 650 & 750 & 700 & 800 \\
\hline & 2 & & 1 & 2 & 700 & 800 & 750 & 850 \\
\hline & 2 & & 2 & 1 & 600 & 700 & 650 & 750 \\
\hline & 2 & & 2 & 2 & 550 & 650 & 600 & 700 \\
\hline \multirow[t]{4}{*}{$\mathrm{USP}_{p d}^{i}$} & 1 & 1 & 1 & & 850 & 950 & 900 & 1000 \\
\hline & 1 & 1 & 2 & & 750 & 850 & 800 & 900 \\
\hline & 2 & 1 & 1 & & 400 & 500 & 450 & 550 \\
\hline & 2 & 1 & 2 & & 300 & 400 & 350 & 450 \\
\hline \multirow[t]{4}{*}{$\mathrm{USP}_{r c}^{i}$} & 1 & & & 1 & 1650 & 1750 & 1700 & 1800 \\
\hline & 1 & & & 2 & 1600 & 1700 & 1650 & 1750 \\
\hline & 2 & & & 1 & 1000 & 1100 & 1050 & 1150 \\
\hline & 2 & & & 2 & 950 & 1050 & 1000 & 1100 \\
\hline
\end{tabular}

Table 3

Parameters for defining fuzzy product prices in illustrative example

the fluctuating rate for cost parameters. The whole scheduling horizon is five periods. The product demand scenarios and the assigned probabilities are shown in Table 1 for the case study. Other indices and sets are $[\mathcal{N}]=3,[\mathcal{K}]=3$, and $\left[\mathcal{K}^{\prime}\right]=3$. Values of all cost parameters are listed in Tables 2 and 3, and other parameters, Table 4. Notably, Table 3 gives

\begin{tabular}{|c|c|c|c|c|c|c|c|c|c|}
\hline \multicolumn{3}{|c|}{ Trans. Lead Time } & \multicolumn{4}{|c|}{ Safe Inven Quant } & \multicolumn{3}{|c|}{ Trans. Cap. Level } \\
\hline \multicolumn{3}{|c|}{$p d r$} & \multicolumn{4}{|c|}{$i p d r$} & \multicolumn{3}{|c|}{$k p d r$} \\
\hline & 11 & 0 & & 1 & 1 & 150 & & $\begin{array}{lll}1 & 1 & 1\end{array}$ & 60 \\
\hline \multirow[t]{3}{*}{$\mathrm{TLT}_{d r}$} & 12 & & $\operatorname{SIQ}_{r}^{i}$ & 1 & 2 & 150 & & 11 & 120 \\
\hline & 21 & 1 & & 2 & 1 & 250 & & 11 & 180 \\
\hline & 22 & 1 & & 2 & 2 & 250 & & 12 & 60 \\
\hline \multicolumn{2}{|c|}{$\mathrm{TLT}_{p d} 11$} & 0 & & 1 & 1 & 200 & & 12 & 120 \\
\hline \multirow{2}{*}{\multicolumn{2}{|c|}{$\frac{12}{\text { Max Invent }}$}} & 1 & $\operatorname{SIQ}_{d}^{i}$ & 1 & 2 & $300^{\circ}$ & $\mathrm{TCL}_{d r}^{k}$ & 12 & 180 \\
\hline & & Max Inventory Cap & & 2 & 1 & 300 & & 21 & 220 \\
\hline \multicolumn{3}{|c|}{$p d r$} & & 2 & 2 & 500 & & 21 & 440 \\
\hline \multirow[t]{2}{*}{$\mathrm{MIC}_{r}$} & 1 & 600 & $\mathrm{SIQ}_{p}^{i}$ & 11 & & 400 & & 21 & 660 \\
\hline & 2 & 800 & & 21 & & 600 & & 22 & 220 \\
\hline \multirow[t]{2}{*}{$\mathrm{MIC}_{d}$} & 1 & 1000 & Fix M & $\mathrm{anu}$ & Que & ntity & & 22 & 440 \\
\hline & 2 & 4000 & & $i 1$ & & & & $\begin{array}{ll}3 & 22 \\
\end{array}$ & 660 \\
\hline \multicolumn{2}{|c|}{$\mathrm{MIC}_{p} 1$} & 2500 & $\mathrm{FMQ}_{p}^{i}$ & 11 & & 1200 & & 111 & 100 \\
\hline \multicolumn{3}{|c|}{ Total Overtime \# } & & 21 & & 1500 & & 211 & 200 \\
\hline \multicolumn{2}{|c|}{$p$} & & $\mathrm{OMQ}_{p}^{i}$ & 11 & & $300^{\prime}$ & $\mathrm{TCL}_{p d}^{k^{\prime}}$ & 311 & 300 \\
\hline \multirow{7}{*}{\multicolumn{2}{|c|}{$\mathrm{MTO}_{p} 1$}} & 3 & & 21 & & 400 & & 112 & 400 \\
\hline & & & & & & & & 212 & 800 \\
\hline & & & & & & & & 312 & 1200 \\
\hline & & & $\operatorname{Max}$ & $m_{2}$ & it $/ \mathrm{O}$ & atput $T$ & ranspor & t Capaci & ties \\
\hline & & & & & $d$ & & & $d$ & \\
\hline & & & $\mathrm{MITC}_{d}$ & & 1 & 300 & MOTC $_{d}$ & 1 & 300 \\
\hline & & & & & 2 & 1200 & & 2 & 1200 \\
\hline
\end{tabular}
the required parameters for defining fuzzy product prices

Table 4

Other parameters of illustrative example 
Table 5

Parameters for defining membership functions for objectives

\begin{tabular}{|c|c|c|c|c|c|}
\hline$m$ & $J_{m}$ & $\underline{J}_{m}^{0}$ & $J_{m}^{0}$ & $J_{m}^{1}$ & $\bar{J}_{m}^{1}$ \\
\hline 1 & $Z_{p=1}$ & $2,273,758$ & $2,273,758$ & $2,664,123$ & $4,623,672$ \\
\hline 2 & $Z_{d=1}$ & $-290,481$ & 37,429 & 570,869 & $1,104,309$ \\
\hline 3 & $Z_{d=2}$ & $-467,993$ & 762,862 & $1,109,662$ & $3,155,891$ \\
\hline 4 & $Z_{r=1}$ & $-797,333$ & 28,323 & 639,630 & $1,345,449$ \\
\hline 5 & $Z_{r=2}$ & $-755,271$ & 276,206 & 648,639 & $1,553,022$ \\
\hline 6 & $\mathrm{SIL}_{p=1}$ & 0.09 & 0.09 & 0.92 & 0.92 \\
\hline 7 & $\mathrm{SIL}_{d=1}$ & 0.07 & 0.07 & 0.97 & 0.97 \\
\hline 8 & $\mathrm{SIL}_{d=2}$ & 0.02 & 0.02 & 0.95 & 0.95 \\
\hline 9 & $\mathrm{SIL}_{r=1}$ & 0.06 & 0.06 & 0.94 & 0.94 \\
\hline 10 & $\mathrm{SIL}_{r=2}$ & 0.04 & 0.04 & 0.93 & 0.93 \\
\hline 11 & $\mathrm{CSL}_{r=1}$ & 0.80 & 0.80 & 1.00 & 1.00 \\
\hline 12 & $\mathrm{CSL}_{r=2}$ & 0.77 & 0.77 & 0.96 & 0.96 \\
\hline 13 & $\mathrm{RI}_{Z_{p=1}}$ & $-204,289$ & $-129,519$ & & \\
\hline 14 & $\mathrm{RI}_{Z_{d=1}}$ & $-101,687$ & $-75,487$ & & \\
\hline 15 & $\mathrm{RI}_{Z_{d=2}}$ & $-193,525$ & $-159,639$ & & \\
\hline 16 & $\mathrm{RI}_{Z_{r=1}}$ & $-200,097$ & $-117,435$ & & \\
\hline 17 & $\mathrm{RI}_{Z_{r=2}}$ & $-244,216$ & $-139,514$ & & \\
\hline 18 & $\mathrm{RI}_{\mathrm{SIL}_{p=1}}$ & -0.066 & -0.048 & & \\
\hline 19 & $\mathrm{RI}_{\mathrm{SIL}_{d=1}}$ & -0.073 & -0.060 & & \\
\hline 20 & $\mathrm{RI}_{\mathrm{SIL}_{d=2}}$ & -0.057 & -0.039 & & \\
\hline 21 & $\mathrm{RI}_{\mathrm{SIL}_{r=1}}$ & -0.086 & -0.034 & & \\
\hline 22 & $\mathrm{RI}_{\mathrm{SIL}_{r=2}}$ & -0.085 & -0.066 & & \\
\hline 23 & $\mathrm{RI}_{\mathrm{CSL}_{r=1}}$ & -0.036 & -0.017 & & \\
\hline 24 & $\mathrm{RI}_{\mathrm{CSL}_{r=2}}$ & -0.026 & -0.019 & & \\
\hline
\end{tabular}

for sellers and buyers, respectively. Membership functions shown in Fig. 3 are respectively adopted for all aggregated fuzzy product prices.

The problem includes 5702 equations, 4079 continuous variables, and 910 binary variables. To solve this mixed-integer nonlinear programming problem for the supply chain model, the Generalized Algebraic Modeling System (GAMS, Brooke et al., 1998), a well-known high-level modeling system for mathematical programming problems,
Table 6

The compromised product prices of the illustrative example

\begin{tabular}{llllllll}
\hline & $i$ & $p$ & $d$ & $r$ & USP & $\mu_{\mathcal{B P}}$ & $\mu_{\mathcal{S P}}$ \\
\hline $\mathrm{USP}_{d r}^{i}$ & 1 & & 1 & 1 & 1425 & 0.75 & 0.75 \\
& 1 & & 1 & 2 & 1475 & 0.75 & 0.75 \\
& 1 & & 2 & 1 & 1326 & 0.74 & 0.76 \\
& 1 & & 2 & 2 & 1276 & 0.75 & 0.75 \\
& 2 & & 1 & 1 & 725 & 0.75 & 0.75 \\
& 2 & & 1 & 2 & 775 & 0.75 & 0.75 \\
& 2 & & 2 & 1 & 676 & 0.74 & 0.76 \\
& 2 & & 2 & 2 & 626 & 0.74 & 0.76 \\
$\mathrm{USP}_{p d}^{i}$ & 1 & 1 & 1 & & 926 & 0.74 & 0.76 \\
& 1 & 1 & 2 & & 826 & 0.75 & 0.75 \\
& 2 & 1 & 1 & & 476 & 0.74 & 0.76 \\
& 2 & 1 & 2 & & 375 & 0.75 & 0.75 \\
$\mathrm{USP}_{r c}^{i}$ & 1 & & & 1 & 1728 & 0.72 & 0.78 \\
& 1 & & & 2 & 1678 & 0.72 & 0.78 \\
& 2 & & & 1 & 1079 & 0.72 & 0.78 \\
& 2 & & & 2 & 1031 & 0.69 & 0.81 \\
\hline
\end{tabular}

is used as the solution environment. The MINLP solver used is DICOPT and the NLP solver, CONOPT.

According to the problem description, mathematical formulation, and parameter design mentioned previously, we solve the multi-objective mixed-integer non-linear programming problem for a production and distribution scheduling by using the fuzzy procedure discussed in Section 5 .

Step 1. Select suitable ranges for defining membership functions. Relevant lower/upper limits, $\underline{J}_{m}^{0}$ and $\bar{J}_{m}^{1}$, and selected effective ranges, $\left[J_{m}^{0}, J_{m}^{1}\right]$, for membership functions are shown in Table 5. As mentioned previously, one can subjectively select values for $J_{m}^{0}$ and $J_{m}^{1}$ for each objective if meaningful lower/upper bounds can be expected.
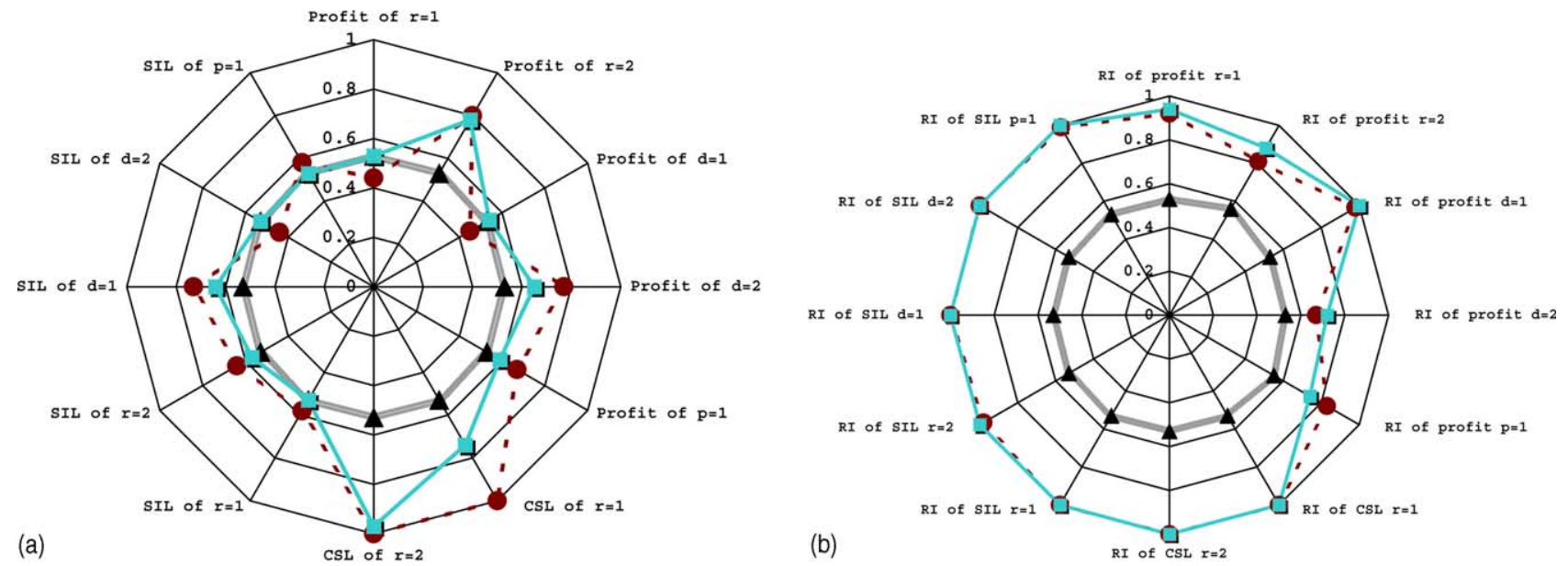

$\Delta \quad \mathrm{T}$-norm $=\min \quad-\mathrm{T}$-norm $=$ product

- Two phase method

Fig. 5. Radar plots by using minimum or product $t$-norms (single-phase optimization) and proposed two-phase optimization method. (a) Objectives such as profits, inventory levels, and service levels; (b) robustness measures. 
Table 7

Variability of scenario-dependent objective values

\begin{tabular}{|c|c|c|c|c|c|c|c|c|c|c|c|c|c|}
\hline & \multirow[t]{2}{*}{ Scenario } & \multicolumn{5}{|l|}{ Profit } & \multicolumn{5}{|c|}{ Safe inventory level } & \multicolumn{2}{|c|}{ Service level } \\
\hline & & $p=1$ & $d=1$ & $d=2$ & $r=1$ & $r=2$ & $p=1$ & $d=1$ & $d=2$ & $r=1$ & $r=2$ & $r=1$ & $r=2$ \\
\hline \multirow[t]{7}{*}{ RI not included } & 1 & 2709353 & 353342 & 1300455 & 657240 & 288886 & 0.60 & 0.64 & 0.43 & 0.60 & 0.53 & 1.00 & 1.00 \\
\hline & 2 & 2036686 & 413862 & 821158 & 852790 & 917316 & 0.47 & 0.82 & 0.50 & 0.74 & 0.53 & 1.00 & 1.00 \\
\hline & 3 & 2806934 & 250369 & 1196849 & 79628 & 736006 & 0.49 & 0.71 & 0.57 & 0.50 & 0.62 & 0.99 & 1.00 \\
\hline & 4 & 2447388 & 375178 & 936194 & 234508 & 756312 & 0.61 & 0.97 & 0.57 & 0.59 & 0.75 & 0.99 & 0.85 \\
\hline & 5 & 2648390 & 421579 & 1159809 & 210789 & -271410 & 0.65 & 0.84 & 0.55 & 0.68 & 0.74 & 1.00 & 1.00 \\
\hline & 6 & 2760923 & 123027 & 1053518 & 229115 & 46819 & 0.64 & 0.91 & 0.57 & 0.62 & 0.67 & 1.00 & 1.00 \\
\hline & 7 & 2387742 & 318640 & 772951 & -253262 & 853122 & 0.69 & 0.95 & 0.60 & 0.63 & 0.75 & 1.00 & 0.99 \\
\hline Expected & & 2502362 & 332101 & 1023055 & 366009 & 538010 & 0.58 & 0.84 & 0.53 & 0.62 & 0.65 & 1.00 & 0.96 \\
\hline RI & & -114353 & -37930 & -78733 & -141041 & -152013 & -0.033 & -0.050 & -0.021 & -0.025 & -0.046 & -0.004 & -0.026 \\
\hline \multirow[t]{7}{*}{ RI included } & 1 & 2627463 & 327660 & 987458 & 396451 & 637799 & 0.53 & 0.65 & 0.51 & 0.52 & 0.55 & 0.95 & 0.96 \\
\hline & 2 & 2580353 & 327660 & 1034208 & 350482 & 595791 & 0.53 & 0.65 & 0.51 & 0.52 & 0.55 & 0.95 & 0.96 \\
\hline & 3 & 2502549 & 327660 & 1151319 & 350482 & 565329 & 0.53 & 0.65 & 0.51 & 0.52 & 0.55 & 0.95 & 0.96 \\
\hline & 4 & 2502549 & 327660 & 1050643 & 350482 & 565329 & 0.53 & 0.65 & 0.51 & 0.52 & 0.55 & 0.95 & 0.96 \\
\hline & 5 & 2485710 & 327660 & 817240 & 299611 & 452736 & 0.53 & 0.65 & 0.51 & 0.52 & 0.55 & 0.95 & 0.96 \\
\hline & 6 & 2451158 & 327660 & 765471 & 332659 & 528373 & 0.53 & 0.65 & 0.51 & 0.52 & 0.55 & 0.95 & 0.96 \\
\hline & 7 & 2129671 & 327660 & 987458 & 350482 & 536394 & 0.53 & 0.65 & 0.51 & 0.52 & 0.55 & 0.95 & 0.96 \\
\hline Expected & & 2502549 & 327660 & 987458 & 350482 & 565329 & 0.53 & 0.65 & 0.51 & 0.52 & 0.55 & 0.95 & 0.96 \\
\hline RI & & -34298 & 0 & -44178 & -6895 & -16963 & 0.00 & 0.00 & 0.00 & 0.00 & 0.00 & 0.00 & 0.00 \\
\hline
\end{tabular}

We thus directly use $\left[\underline{J}_{m}^{0}, \bar{J}_{m}^{1}\right]$ as the effective range for defining fuzzy objectives such as inventory levels and customer service levels. Due to the wide variability of scenario-dependent profits, it is suggested using the lowest positive profit as the lower bound, and the second largest value as the upper bound. For emphasizing robustness measures, we suggest adopting the second lower value as the lower bound, and the zero as the upper bound.

Step 2 (Phase I). To maximize the degree of satisfaction for the worst objective by using the minimum operator. The result is $\mu_{\min }=0.53$.

Step 3 (Phase II). Re-optimize the problem with new constraints of guaranteed minimum satisfaction for all fuzzy objectives and fuzzy product prices. The results will be shown and discussed in the following.

The radar plots for profits, safe inventory levels, customer service levels, and the robustness measures are shown in Fig. 5, and the resulting compromised sales prices are listed in Table 6.

Form the results obtained by selecting minimum as $t$-norm, we can get a more balanced satisfaction among all objectives where the degrees of satisfaction are all around 0.53 . By using product operator to guarantee a unique solution, however, the results are unbalanced with lower degree of satisfaction for profits of $r=1$ and $d=1$, and the safe inventory level of $d=2$. On the other hand, the high profit of $r=2$ and service levels of $r=1$ and $r=2$ are given very high emphasis. Obviously this is not desirable for obtaining a compromise solution. Overcoming the drawbacks of the single phase method, the proposed two-phase method can incorporate advantages of these two $t$-norms. The minimum operator is used in phase I to find the maximal satisfaction for worst situation, and the product operator is applied in phase II to maximize the overall satisfaction with guaranteed minimal fulfillment for all fuzzy objectives and fuzzy product prices.

Resulting objective values for all scenarios either considering robustness measures or not are listed in Table 7. It can be found that the variability of scenario-dependent objective values is quite high if the robustness measures are not included as objectives. In such a case, some profit realizations are unacceptably low for certain scenarios especially for retailers $r=1$ and $r=2$. When simultaneously considering the robustness measures as objectives, i.e., objective values less than the expectation are penalized, the variability of scenario-related objective values is significantly reduced.

\section{Conclusion}

This paper investigates the simultaneous optimization of multiple conflict objectives and the uncertain product prices problem in a typical supply chain network with market demand uncertainties. The demand uncertainty is modeled as discrete scenarios with given probabilities for different expected outcomes, and the uncertain product prices are described as fuzzy variables. The problem is formulated as a MINLP model to achieve fair profit distribution among whole network's participants, safe inventory levels, maximum customer service levels, maximum robustness to 
demand uncertainties, and to guarantee maximum acceptability levels of sellers' and buyers' preference on product prices. Considering the robustness measures as part of multiple objectives can significantly reduce the variability of other objective values to product demand uncertainties. To find the degree of satisfaction of the multiple objectives, the linear increasing membership function is used; the final decision is acquired by fuzzy aggregation of the fuzzy goals and the fuzzy product prices, and the best compromised solution can be derived by maximizing the overall degree of satisfaction for the decision. The implementation of the proposed fuzzy decision-making method, as one can see in the case study, demonstrates that the method can provide a compensatory solution for the multiple conflict objectives and the fuzzy product prices problem in a supply chain network with demand uncertainties.

\section{Nomenclature}

\begin{tabular}{|c|c|c|}
\hline $\begin{array}{l}\text { Index/set } \\
c \in \mathcal{C} \\
d \in \mathcal{D} \\
i \in \mathcal{I} \\
k \in \mathcal{K} \\
k^{\prime} \in \mathcal{K}^{\prime} \\
m \in \mathcal{M} \\
m^{\prime} \in \mathcal{M}^{\prime} \\
n \in \mathcal{N} \\
p \in \mathcal{P} \\
r \in \mathcal{R} \\
t \in \mathcal{T} \\
s \in \mathcal{S}\end{array}$ & $\begin{array}{l}\text { Dimension } \\
{[\mathcal{C}]=C} \\
{[\mathcal{D}]=D} \\
{[\mathcal{T}]=I} \\
{[\mathcal{K}]=K} \\
{\left[\mathcal{K}^{\prime}\right]=K^{\prime}} \\
{[\mathcal{M}]=M} \\
{\left[\mathcal{M}^{\prime}\right]=M^{\prime}} \\
{[\mathcal{N}]=N} \\
{[\mathcal{P}]=P} \\
{[\mathcal{R}]=R} \\
{[\mathcal{T}]=T} \\
{[\mathcal{S}]=S}\end{array}$ & $\begin{array}{l}\text { Physical meaning } \\
\text { customers } \\
\text { distribution centers } \\
\text { products } \\
\text { transport capacity level, DC to retailer } \\
\text { transport capacity level, plant to DC } \\
\text { all objectives } \\
\text { objectives } 1-3 \\
\text { counter for overtime manufacturing } \\
\text { plants } \\
\text { retailers } \\
\text { periods } \\
\text { scenarios }\end{array}$ \\
\hline $\begin{array}{l}\text { Parameters } \\
\mathrm{FCD}_{* t s}^{i} \\
\mathrm{FIC}_{*}^{i} \\
\mathrm{FMC}_{*}^{i} \\
\mathrm{FMQ}_{*}^{i} \\
\mathrm{FTC}_{*}^{k} \\
\mathrm{FTC}_{*}^{k^{\prime}} \\
\mathrm{MIC}_{*} \\
\mathrm{MITC}_{*} \\
\mathrm{MOTC}_{*} \\
\text { MTO }_{*} \\
\mathrm{OMC}_{*}^{i} \\
\mathrm{OMQ}_{*}^{i} \\
\operatorname{PPD}_{*} \\
\mathrm{SIQ}_{*}^{i} \\
\mathrm{SQL}_{*}^{i \vee} \\
\mathrm{UHC}_{*}^{i} \\
\mathrm{UIC}_{*}^{i} \\
\mathrm{UMC}_{*}^{i} \\
\left(\mathrm{USP}_{*}^{i}\right)_{+}^{\bullet} \\
\mathrm{UTC}_{*}^{k} \\
\mathrm{UTC}_{*}^{k^{\prime}} \\
\operatorname{TCL}_{*}^{k} \\
\operatorname{TCL}_{*}^{k^{\prime}} \\
\operatorname{TLT}_{*}\end{array}$ & $\begin{array}{l}* \in \\
\{r\} \\
\{p\} \\
\{p\} \\
\{p\} \\
\{d r\} \\
\{p d\} \\
\{p, d, r\} \\
\{d\} \\
\{d\} \\
\{p\} \\
\{p\} \\
\{p\} \\
\{s\} \\
\{p, d, r\} \\
\{p, d\} \\
\{p, d, r\} \\
\{p, d, r\} \\
\{p\} \\
\{p d, d r, r c\} \\
\{d r\} \\
\{p d\} \\
\{d r\} \\
\{p d\} \\
\{p d, d r\}\end{array}$ & $\begin{array}{l}\text { Physical meaning } \\
\text { forecast customer demand of } i \\
\text { fix idle cost to keep } p \text { idle } \\
\text { fix manufacture cost changed to make } i \\
\text { fix manufacture quantity of } i \\
k \text { th level fix transport cost, } d \text { to } r \\
k^{\prime} \text { th level fix transport cost, } p \text { to } d \\
\text { maximum inventory capacity of } p, d, r \\
\text { maximum input transport capacity of } d \\
\text { maximum output transport capacity of } d \\
\text { maximum total overtime manufacture period } \\
\text { overtime unit manufacture cost of } i \\
\text { overall fix manufacture quantity } \\
\text { probability for scenario } s \\
\text { safe inventory quantity in } p, d, r \\
\text { sales quantity levels of } i,(*, \vee) \in\left\{\left(p, \ell^{\prime}\right),(d, \ell)\right\} \\
\text { unit handling cost of } i \text { for } p, d, r \\
\text { unit inventory cost of } i \text { for } p, d, r \\
\text { unit manufacture cost of } i \\
\text { parameters for defining piecewise unit sale prices, }+\in\{S, B\}, \bullet \in\{0,1\} \\
k \text { th level unit transport cost, } d \text { to } r \\
k^{\prime} \text { th level unit transport cost, } p \text { to } d \\
k \text { th transport capacity level, } d \text { to } r \\
k^{\prime} \text { th transport capacity level, } p \text { to } d \\
\text { transport lead time, } p \text { to } d(d \text { to } r)\end{array}$ \\
\hline $\begin{array}{l}\text { Binary variables } \\
Y_{* t s}^{k} \\
Y_{* t s}^{k^{\prime}} \\
\alpha_{* t s}^{i} \\
\beta_{* t s}^{i}\end{array}$ & $\begin{array}{l}* \in \\
\{d r\} \\
\{p d\} \\
\{p\} \\
\{p\}\end{array}$ & $\begin{array}{l}\text { Meaning when having value of } 1 \\
k \text { th transport capacity level, } d \text { to } r \\
k^{\prime} \text { th transport capacity level, } p \text { to } d \\
\text { manufacture with regular time workforce } \\
\text { setup plant } p \text { to manufacture } i\end{array}$ \\
\hline
\end{tabular}




\begin{tabular}{lll}
\hline$\gamma_{* t s}^{i}$ & $\{p\}$ & $p$ changeover to manufacture $i$ \\
$o_{* t s}^{i}$ & $\{p\}$ & manufacture with overtime workforce \\
Real variables & $* \in$ & Physical meaning \\
$B_{* t s}^{i}$ & $\{r\}$ & backlog level of $i$ in $r$ at end of $t$ \\
$D_{* t s}^{i}$ & $\{p, d, r\}$ & short safe inventory level in $p, d, r$ \\
$I_{* t s}^{i}$ & $\{p, d, r\}$ & inventory level of $i$ in $p, d, r$ \\
$J_{*}$ & $\{m\}$ & objectives \\
$\mathrm{PSP}_{* t s}$ & $\{p, d, r\}$ & product sales of $p, d, r$ \\
$\mathrm{RI}_{*}$ & $\left\{m^{\prime}\right\}$ & robustness index of objectives \\
$\mathrm{SQ}_{* t s}^{i}$ & $\{p d, d r, r c\}$ & sales quantity of $i$ from $p$ to $d, d$ to $r, r$ to $c$ \\
$\mathrm{TIC}_{* t s}$ & $\{p, d, r\}$ & total inventory cost of $p, d, r$ \\
$\mathrm{THC}_{* t s}$ & $\{p, d, r\}$ & total handling cost of $p, d, r$ \\
$\mathrm{TMC}_{* t s}$ & $\{p\}$ & total manufacture cost of $p$ \\
$\mathrm{TPC}_{* t s}$ & $\{d, r\}$ & total purchase cost of $d, r$ \\
$\mathrm{TQ}_{* t s}^{k}$ & $\{d r\}$ & $k$ th level transport quantity, $d$ to $r$ \\
$\mathrm{TQ}_{* t s}^{k^{\prime}}$ & $\{p d\}$ & $k^{\prime}$ th level transport quantity, $p$ to $d$ \\
$\mathrm{TQ}_{* t s}$ & $\{p d, d r\}$ & total transport quantity, $p$ to $d$ or $d$ to $r$ \\
$\mathrm{TTC}_{* t s}$ & $\{d ; p d, d r\}$ & total transport cost of $d ; p$ to $d$ or $d$ to $r$ \\
$\mathrm{USP}_{* s}^{i}$ & $\{p d, d r, r c\}$ & unit product price of $i, p$ to $d, d$ to $r$, and $r$ to $c$ \\
$\mathrm{SIL}_{* t}$ & $\{p, d, r\}$ & expected safe inventory level of $p, d, r$ \\
$\mathrm{CSL}_{* t}$ & $\{r\}$ & expected customer service level of $r$ \\
$Z_{* t}$ & $\{p, d, r\}$ & expected net profit of $p, d, r$ \\
$F u z z y$ variables & $* \in$ & Physical meaning \\
$\mathcal{B} \mathcal{P}_{*}^{i}$ & $\{p d, d r, r c\}$ & fuzzy sets to measure buyer's preference for product price \\
$\mathcal{F} \mathcal{D}^{i}$ & $\{p d, d r, r c\}$ & fuzzy set for final decision \\
$\mathcal{J}_{m}$ & & fuzzy set for objective $m, m \in \mathcal{M}$, \\
$\mathcal{S P}_{*}^{i}$ & fuzzy sets to measure seller's preference for product price \\
\hline & &
\end{tabular}

\section{Acknowledgements}

This work was supported by the National Science Council (ROC) under Contract NSC91-2214-E-002-001.

\section{References}

Ahmed, S., \& Sahinidis, N. V. (1998). Robust process planning under uncertainty. Industrial Engineering in Chemical Research, 37, 1883.

Applequist, G. E., Pekny, J. F., \& Reklaitis, G. V. (2000). Risk and uncertainty in managing chemical manufacturing supply chains. Computer and Chemical Engineering, 24, 2211.

Bose, S., \& Pekny, J. F. (2000). A model predictive framework for planning and scheduling problems: A case study of customer goods supply chain. Computer and Chemical Engineering, 24, 329.

Brooke, A., Kendrick, D., Meeraus, A., Raman, R., \& Rosenthal, R. E. (1988). GMAS: A user's guide. GAMS Development Corporation.

Chen, C. L., Wang, B. W., \& Lee, W. C. (2003). Multi-objective optimization for a multi-enterprise supply chain network. Industrial Engineering in Chemical Research, 42, 1879.

Cheng, L., Subrahmanian, E., \& Westerberg, A. W. (2003). Design and planning under uncertainty: Issues on problem formulation and solution. Computer and Chemical Engineering, 27, 781.

Delgado, M., Herrera, F., \& Verdegay, J. L. (1993). Post-optimality analysis on the membership function of a fuzzy linear programming problem. Fuzzy Sets and Systems, 53, 289.

Garcia-Flores, R., Wang, X. Z., \& Goltz, G. E. (2000). Agent-based information flow for process industries' supply chain modeling. Computer and Chemical Engineering, 24, 1135.

Giannoccaro, I., Pontrandolfo, P., \& Scozzi, B. (2003). A fuzzy echelon approach for inventory management in supply chains. European Journal of Operational Research, 149, 185.

Gjerdrum, J., Shah, N., \& Papageorgiou, L. G. (2001). Transfer price for multi-enterprise supply chain optimization. Industrial Engineering in Chemical Research, 40, 1650.

Gupta, A., \& Maranas, C. D. (2000). A two-stage modeling and solution framework for multi-site midterm planning under demand uncertainty. Industrial Engineering in Chemical Research, 39, 3799.

Gupta, A., \& Maranas, C. D. (2003). Managing demand uncertainty in supply hain planning. Computer and Chemical Engineering, 27, 1219.

Gupta, A., Maranas, C. D., \& McDonald, C. M. (2000). Mid-term supply chain planning under demand uncertainty: Customer demand satisfaction and inventory management. Computer and Chemical Engineering, $24,2613$.

Klir, G. L., \& Yuan, B. (1995). Fuzzy sets and fuzzy logics: Theory and application. New York: Prentice Hall.

Li, R. J., \& Lee, E. S. (1993). Fuzzy Multiple objective programming and compromise programming with Pareto optimum. Fuzzy Sets and Systems, 53, 275.

Liu, M. L., \& Sahinidis, N. V. (1997). Process planning in a fuzzy environment. European Journal of Operational Research, 100, 142. 
Perea-Lopez, E., Grossmann, I. E., \& Ydstie, B. E. (2000). Dynamic modeling and decentralized control of supply chains. Industrial Engineering in Chemical Research, 39, 3369.

Petrovic, D., Roy, R., \& Petrovic, R. (1998). Modeling and simulation of a supply chain in an uncertain environment. European Journal of Operational Research, 109, 299.

Petrovic, D., Roy, R., \& Petrovic, R. (1999). Supply chain modeling using fuzzy sets. International Journal of Production Economics, 59, 443.

Pinto, J. M., Joly, M., \& Moro, L. F. L. (2000). Planning and scheduling models for refinery operations. Computer and Chemical Engineering, 24, 2259.

Sakawa, M. (1993). Fuzzy sets and interactive multi-objective optimization. New York: Plenum Press.
Suh, M. H., \& Lee, T. Y. (2001). Robust optimization method for the economic term in chemical process design and planning. Industrial Engineering in Chemical Research, 40, 5950.

Tsiakis, P., Shah, N., \& Pantelides, C. C. (2001). Design of multi-echelon supply chain networks under demand uncertainty. Industrial Engineering in Chemical Research, 40, 3585.

Zadeh, L. A. (1965). Fuzzy sets. Information and Control, 8, 338.

Zhou, Z., Cheng, S., \& Hua, B. (2000). Supply chain optimization of continuous process industries with sustainability considerations. Computer and Chemical Engineering, 24, 1151.

Zimmermann, H. J. (1978). Fuzzy programming and linear programming with several objective functions. Fuzzy Sets and Systems, 1, 45. 